Hydroécol. Appl. (1994) Tome 6 Vol 1-2, pp. 139-151

\title{
Evolution temporelle et spatiale du phytoplancton dans le réservoir de Pareloup
}

\section{Spatial and temporal evolution of phytoplankton in Pareloup reservoir}

\author{
R. Le Cohu, J. Brabet, J. Guitard \& N. Comoy
}

Laboratoire d'Hydrobiologie - ERS 103 C.N.R.S. - Université Paul Sabatier, 118 route de Narbonne, 31062 Toulouse Cedex, France.

Résumé. - L'établissement de la stratification thermique estivale est l'élément dominant du fonctionnement du réservoir de Pareloup: chaque année, elle détermine la mise en place d'une succession phytoplanctonique cyclique. La période de stratification estivale est marquée par une grande diversité spécifique représentée par des espèces de petite taille (rapport $S / N$ élevé); la production primaire est maximale mais la biomasse est faible, le développement des algues étant contrôlé par la prédation. Avec le brassage automnal, on observe une faible augmentation des valeurs de la biomasse; elle est principalement due à la présence de Gonyostomum semen et à un faible regain des diatomées. L'augmentation de la valeur de la biomasse (chlorophylle a) se poursuit tout au long de l'hiver avec le développement de Aulacoseira subarctica. A partir du mois de février, Asterionella formosa prend le relais et constitue le maximum de biomasse du printemps; le déclin d'Asterionella formosa est provoqué par une carence en silice. Une phase d'eaux claires résulte de la sédimentation des diatomées et elle se poursuit pendant tout l'été.

Mots clés. - Phytoplancton, biomasse, succession cyclique.

Abstract. - The establishment of summer stratification is the dominant element in the dynamics of Pareloup reservoir : each year, it determines the start of a cyclic succession of phytoplankton. The period of summer stratification is marked by a high specific diversity in relation with the presence of many small species (high $S N$ ratio); primary production is maximum but the biomass is low, being regulated by predation. With the autumn mix, a small increase in biomass values is observed, due chiefly to the presence of Gonyostomum semen and a slight revival of diatoms. The increase in biomass values (chlorophyll a) continues throughout the winter with the development of Aulacoseira subarctica. Beginning in February, this species is replaced by Asterionella formosa which is the dominant species in the annual spring maximum. Asterionella formosa begins to decline when dissolved silica is depleted. A clear-water phase results from sinking of the diatoms and lasts throughout summer.

Key words. - Phytoplankton, biomass, cyclic succession. 


\section{INTRODUCTION}

La modélisation du fonctionnement de tout écosystème lacustre nécessite la connaissance de la dynamique saisonnière du phytoplancton; celle-ci a été étudiée dans le réservoir de $\mathrm{Pa}$ reloup parallèlement à d'autres approches. Jusqu'à maintenant, dans le Massif central, ce sont surtout les lacs naturels, de faible superficie par rapport à celle du réservoir de Pareloup, qui ont fait l'objet d'études (Devaux 1973 et 1977, Amblard 1988, Amblard et Bourdier, 1990).

Dans ce travail, la périodicité saisonnière de la biomasse (poids frais et chlorophylle) et les variations de la structure du peuplement algal sont analysés. Cette analyse est surtout basée sur les données des années 1986 et 1987 qui reflètent les résultats obtenus sur une dizaine d'années, mais référence sera faite à d'autres années en plusieurs occasions; dans la discussion, les résultats obtenus sont confrontés au modèle P.E.G. proposé par Sommer et al. (1986).

\section{MATÉRIEL ET MÉTHODES}

En 1986 et 1987, des prélèvements hebdomadaires ont été effectués de mars à novembre et c'est pour cette raison que ces deux années nous ont servi de référence. Les autres années, l'échantillonnage a été effectué de façon plus irrégulière sauf en 1991-1992 où nous avons également bénéficié de prélèvements hivernaux. Ils ont été réalisés en un point où la profondeur atteignait $32 \mathrm{~m}$; pendant la période d'isothermie, ils ont porté sur les espaces $0-8,8-16$ et $16-25 \mathrm{~m}$; pendant la période de stratification, ils ont été effectués tous les mètres. A partir de 1987, les algues ont été fixées au glutaraldéhyde neutralisé par le cacodylate de sodium, ce qui a permis une meilleure conservation des flagellés, en particulier des cellules de Gonyostomum semen (Ehr.) Diesing. Les comptages ont été réalisés au microscope inversé (Lund et al. 1958). La densité cellulaire est donnée en nombre de cellules par litre. La biomasse est exprimée d'une part en poids frais $\left(\mu \mathrm{g} . \mathrm{I}^{-1}\right)$ déterminé après estimation du volume des cellules et d'autre part en chlorophylle a.

\section{RÉSULTATS}

\section{Périodicité saisonnière de la biomasse phytoplanctonique}

En 1986 et 1987 (fig. 2), le même modèle de périodicité saisonnière de la biomasse phytoplanctonique se reproduit avec un maximum annuel à la fin du printemps. Ce pic printanier est dû essentiellement aux diatomées et particulièrement à Asterionella formosa Hassal qui peut représenter plus de $90 \%$ de la biomasse. Le développement massif de cette espèce provoque une diminution drastique de la silice (fig. 1), qui devient un facteur limitant $\left(0,31 \mathrm{mg}^{\mathrm{I}^{-1}}\right.$ en $1986,0,17 \mathrm{mg} . \mathrm{l}^{-1}$ 


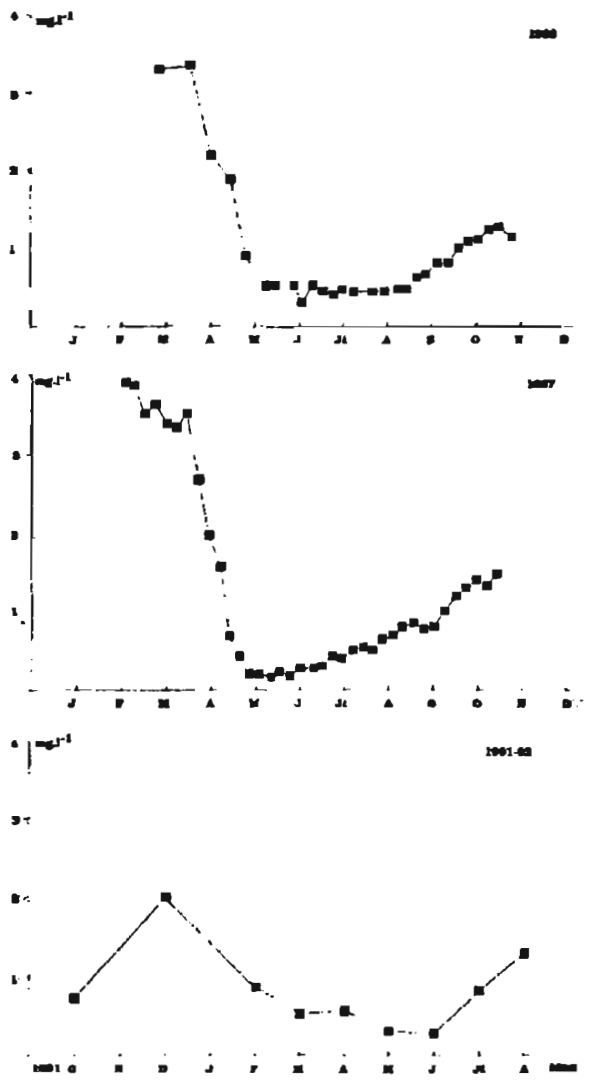

Fig. 1. - Cycle de la silice $\left(\mathrm{SiO}_{2}\right)$.

Fig. 1. - Silica cycle $\left(\mathrm{SiO}_{2}\right)$.

en 1987). Les diatomées sédimentent alors rapidement, ce qui se traduit par une clarification des eaux; la transparence de l'eau mesurée au disque de Secchi passe de 2-3 $\mathrm{m}$ en mars à 6$7 \mathrm{~m}$ en juillet. Cette phase des eaux claires se prolonge pendant toute la période de stratification thermique; elle est marquée par de très faibles valeurs de la biomasse; quelques petits pics apparaissent irrégulièrement : ils sont dus à des poussées fugaces de Gonyostomum semen. Au début de l'automne, le brassage des eaux coïncide avec une augmentation de la densité cellulaire (fig. 2) surtout nette en 1987. Le peuplement algal est alors surtout constitué d'espèces de petite taille qui interfèrent très peu sur les fluctuations de la biomasse. Par exemple, la population de Merismopedia tenuissima Lemm. peut atteindre $12.10^{6}$ cellules par litre le 29/9/87 à $9 \mathrm{~m}$ de profondeur. La biomasse phytoplanctonique est constituée essentiellement par les diatomées (Asterionella formosa, Aulacoseira ambigua (Grun.) Sim. (syn. : Melosira ambigua (Grun.) O. Müll.) et Aulacoseira subarctica (O. Müll.) Haworth (syn. Melosira italica (Ehr.) Kütz. subsp. subarctica O. Müll.) et par Gonyostomum semen.

De mars 1991 à juillet 1992, un suivi mensuel a été réalisé. On retrouve le même schéma général de cycle saisonnier de la biomasse qu'en 1986 et 1987 (fig. 3); cependant, ce qui est très particulier dans le lac de Pareloup, c'est que la phase de croissance de la biomasse débute dès le mois de novembre avec notamment le développement de Aulacoseira subarcti$c a$, ce qui engendre une diminution de la concentration en silice (de $2 \mathrm{mg}^{\mathrm{I}^{-1}}$ à $1 \mathrm{mg}^{-1} \mathrm{I}^{-1}$ ) avant le départ de la croissance d'Asterionella formosa (fig. 2 et 3).

\section{Evolution spatio-temporelle de la chlorophylle a (fig. 4 et 5)}

Les résultats obtenus sur une dizaine d'années, même si les prélèvements n'ont pas été toujours effectués régu- 


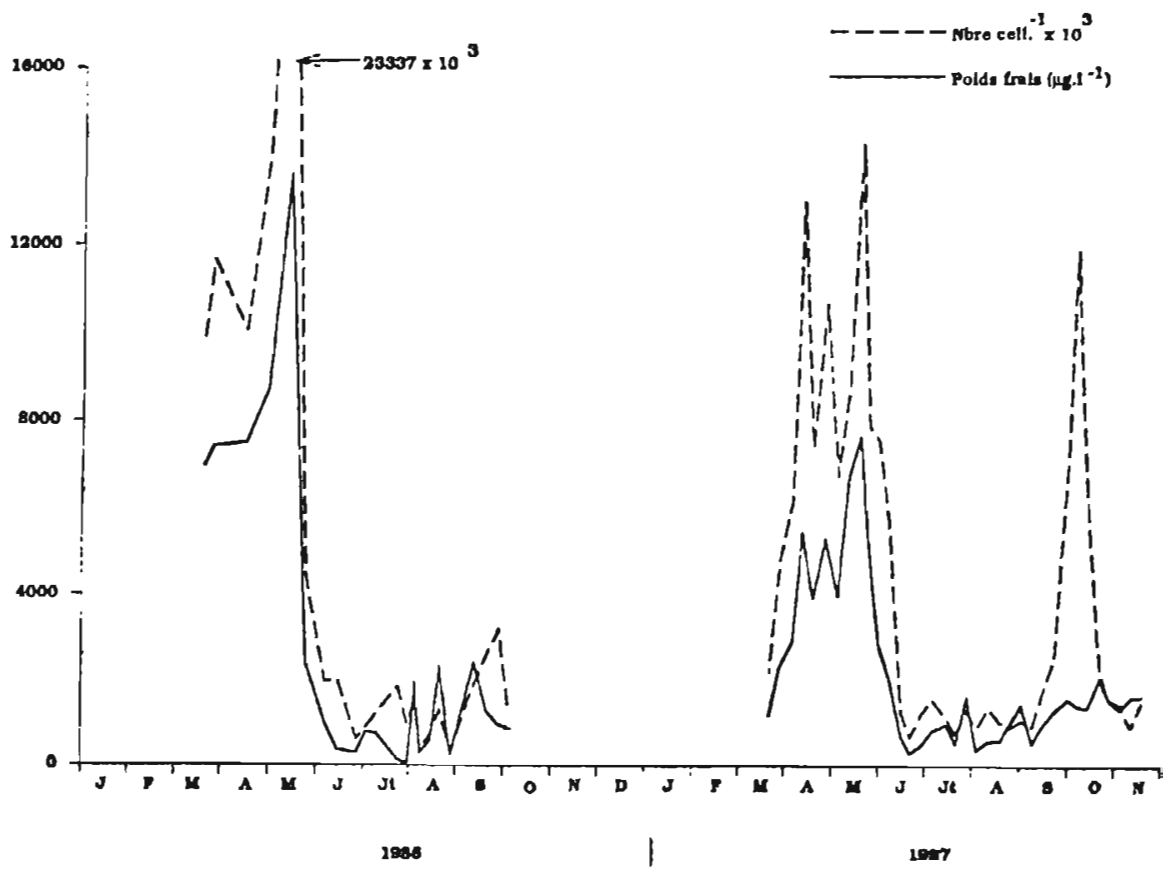

Fig. 2. - Evolution annuelle de la biomasse et de la densité cellulaire en 1986 et 1987.

Fig. 2. - Seasonal fluctuation of the biomass and cell density in 1986 and 1987.

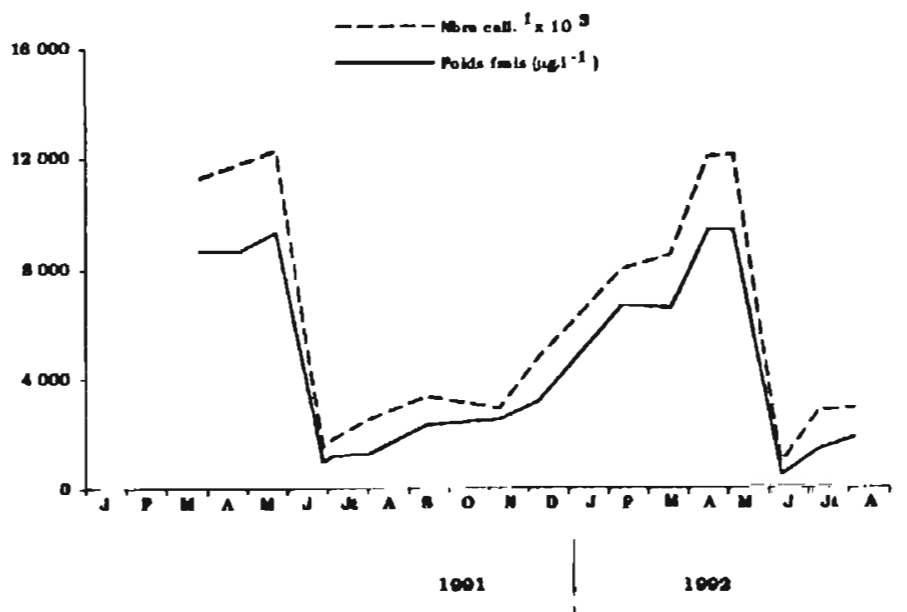

Fig. 3. - Evolution annuelle de la biomasse et de la densité cellulaire en 1991 et 1992.

Fig. 3. - Seasonal fluctuation of the biomass and cell density in 1991 and 1992. 


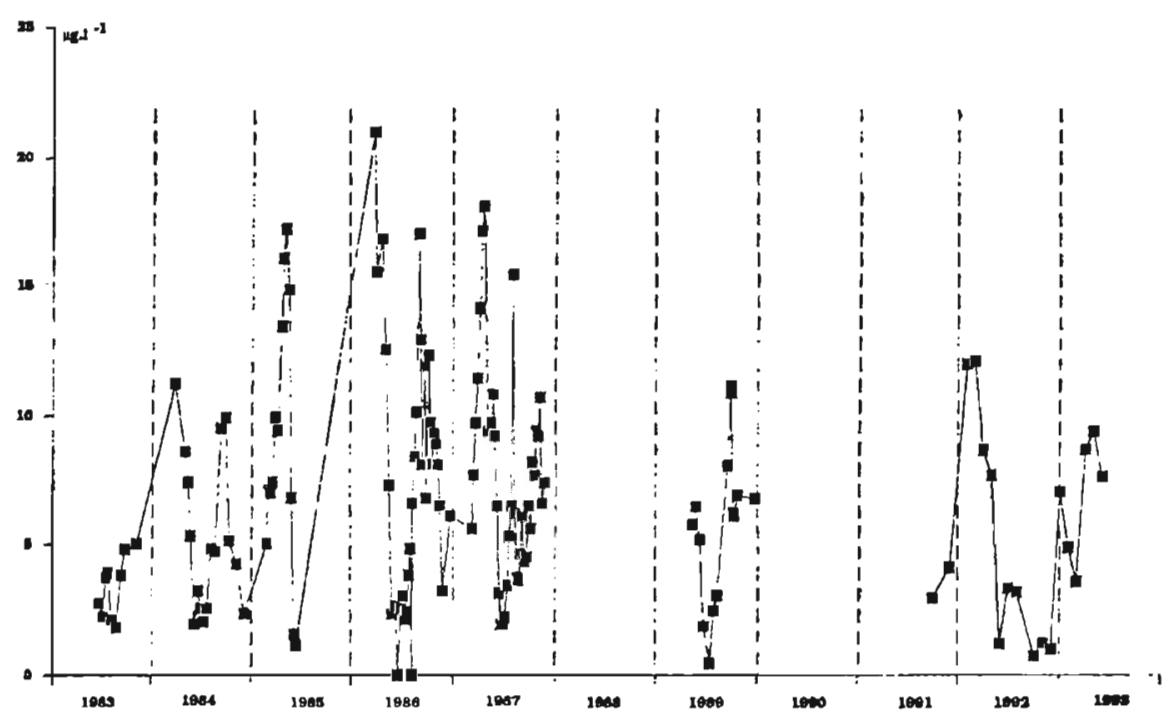

Fig. 4. - Evolution annuelle de la chlorophylle a.

Fig. 4. - Seasonal fluctuation of chlorophyll a.

lièrement, montrent le même type de variations saisonnières, plus ou moins calquées sur celles de la biomasse (en $\mu \mathrm{g} . \mathrm{I}^{-1}$ de poids frais). Si on prend les années de référence 1986 et 1987, les concentrations en chlorophylle a (fig. 4) varient dans une proportion de 1 à 10 en 1986 et de 1 à 6 en 1987. Les valeurs de biomasse ( $\mu$ g. l $^{-1}$ poids frais) montrent des différences plus marquées (fig. 2), de 1 à 29 en 1986 et de 1 à 25 en 1987. Selon Sommer (1986) l'écart entre minimums et maximums de biomasse au sein d'un lac s'amplifie avec le degré de trophie : rapport de 1 à 10 pour les lacs oligotrophes, de 1 à 250 pour les lacs eutrophes. Les variations de ce rapport, les concentrations en chlorophylle a peu élevées et enfin les teneurs en phosphore total (P.T.) de 14-25 $\mu \mathrm{g} . \mathrm{l}^{-1}$ (Sommer et al. 1986) à la fin de l'hiver font que le réservoir de Pareloup peut se ranger dans la catégorie des lacs faiblement mésotrophes.

Au cours de la floraison printanière, un décalage très net apparaît entre les maximums de chlorophylle a (fig. 4, année 1986 et 1987) et de biomasse ou de densité cellulaire (fig. 2). Les quotas cellulaires en chlorophylle a varient de $2 \mathrm{pg}$ à $0,8 \mathrm{pg}$ en 1986 et de $2 \mathrm{pg}$ à $0,5 \mathrm{pg}$ en 1987 . Ces valeurs quoique biaisées du fait qu'elles sont calculées à partir de la densité d'Asterionella formosa, sont proches de celles évaluées par Reynolds (1984) pour cette espèce et confirment les données antérieures dans ce même lac de Pareloup (Jrad 1989).

Dans ce réservoir, les minimums cellulaires en chlorophylle a coïncident avec le maximum de densité cel- 

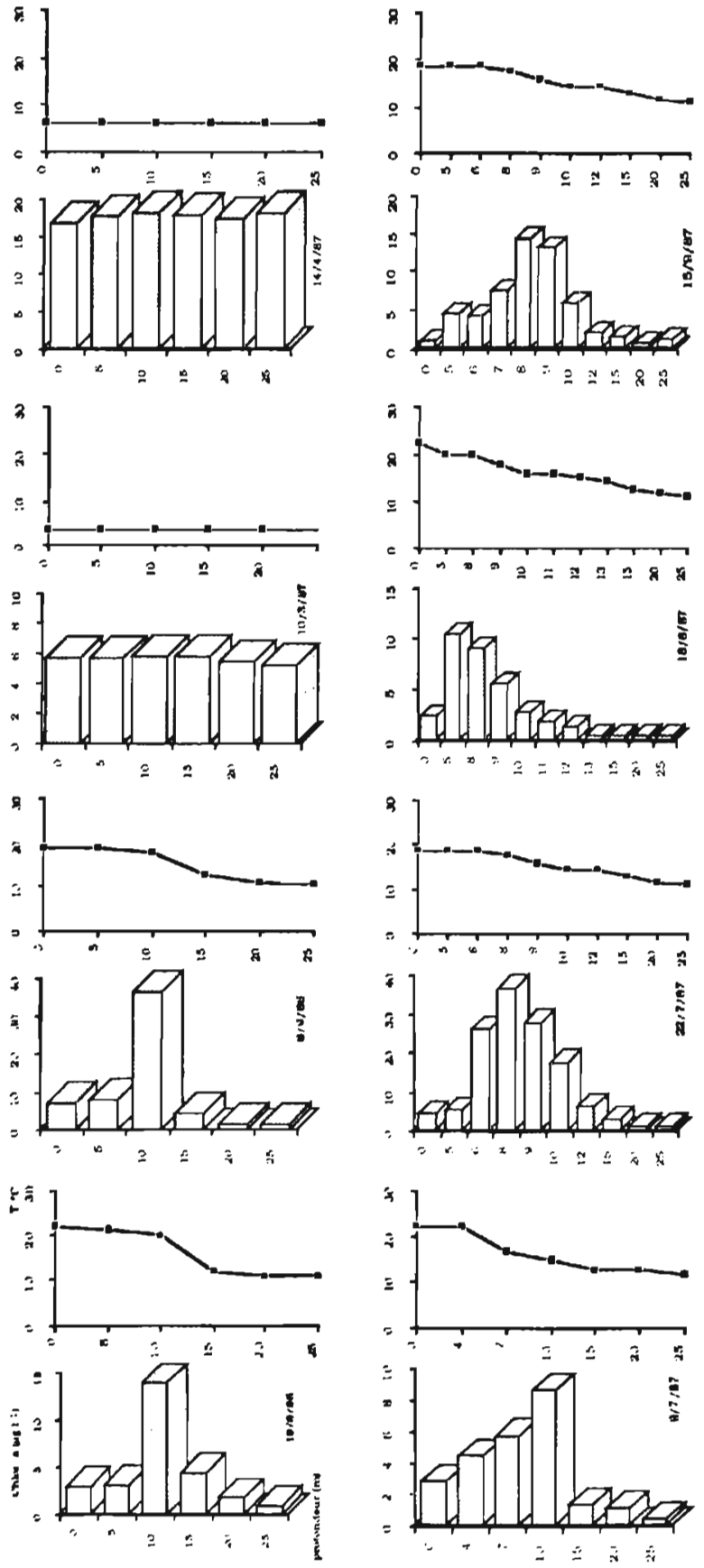

Fig. 5. - Profils verticaux de chlorophylle a et de température dans toute la colonne d'eau explorée. Fig. 5. - Vertical distribution of chlorophyll a and temperature in the whole water column. 
lulaire; ils précèdent de peu le processus de sédimentation. Pendant toute la période de floraison vernale des diatomées, les profils verticaux de la chlorophylle a (fig. 5) sont homogènes sur toute la colonne d'eau. Le temps de séjour des diatomées dans la zone euphotique est alors relativement bref; en général, les algues tendent à compenser le faible éclairement moyen auquel elles sont soumises journellement par une augmentation des quotas cellulaires en chlorophylle a (Falkowski 1984, Hoepffner 1984); ceux-ci ont tendance à diminuer lorsque le jour s'allonge.

Corrélativement à la sédimentation des diatomées, les concentrations en chlorophylle a, comme les valeurs de biomasse, diminuent brutalement juste avant l'installation de la stratification estivale. Pendant toute cette période, les valeurs de la cholorophylle a restent basses, avec le minimum annuel; de temps à autre s'observe un pic comme par exemple en septembre 1986 ou juillet 1987. Ces pics reflètent des discontinuités très marquées de la répartition verticale de la chlorophylle a au sein de la zone euphotique (fig. 5). Le plus souvent, des maximums apparaissent entre 8 et $10 \mathrm{~m}$ de profondeur, juste au dessus ou dans la partie supérieure du métalimnion; ils correspondent (fig. 2) à de courtes périodes de croissance de Gonyostomum se-

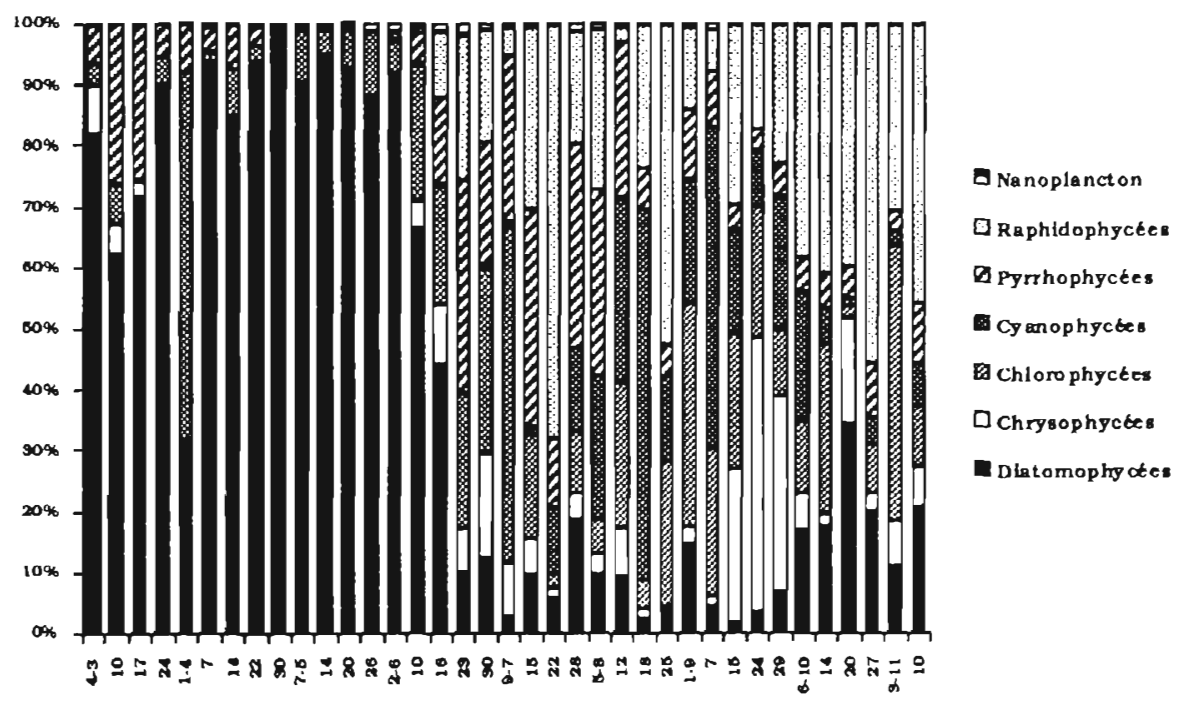

Fig. 6. - Evolution de la structure du peuplement algal (\% de biomasse) au cours de l'année 1987 dans la zone euphotique.

Fig. 6. - Seasonal changes in the relative abundance of the different taxonomic groups $1 \%$ of biomass) in the euphotic zone (1987). 
men (Le Cohu et al. 1989). En fonction de la présence de cette algue les concentrations en chlorophylle a peuvent varier de 1 à $7 \quad\left(5 \mu \mathrm{g} . \mathrm{l}^{-1} \rightarrow\right.$ $35 \mu \mathrm{g} . l^{-1}$ ) suivant les profondeurs.

Lors du suivi 1991-1992, les concentrations maximales annuelles en chlorophylle a se situent en février et mars; comme en 1986 et 1987, on observe un décalage dans le temps entre maximum de chlorophylle a et maximum de biomasse ( $\mu \mathrm{g} . \mathrm{I}^{-1}$ poids frais) ou de densité cellulaire.

\section{Evolution de la structure du peuplement algal}

La figure 6 montre parfaitement la part prépondérante des diatomées dans le pic printanier; le maximum annuel de biomasse (en poids frais) est dû essentiellement à la floraison d'Asterionella formosa qui peut représenter la quasi-totalité de la biomasse. Asterionella formosa est

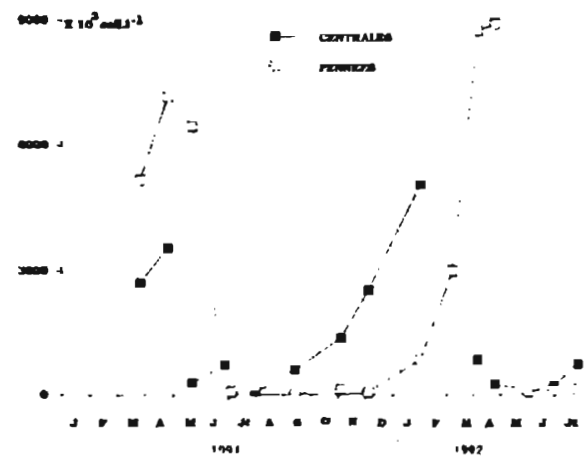

Fig. 7. - Densité cellulaire des diatomées (Aulacoseira sp. et Asterionella formosa) dans la colonne d'eau 0-20 m.

Fig. 7. - Cell density of the diatoms (Aulacoseira sp. and Asterionella formosa) in the water column $0-20 \mathrm{~m}$. accompagnée de quelques centrales : Aulacoseira ambigua, Aulacoseira subarctica, Cyclotella radiosa (Grun.) Lemm. (syn. : Cyclotella comta (Ehr.) Kütz) et Stephanodiscus minutulus (Kütz) Cleve et Möller. Lors du suivi 1991-1992, l'augmentation de biomasse ( $\mu$ g..$^{-1}$ poids frais (fig. 3 ) ou de chlorophylle a, constatée à partir de novembre, est dans un premier temps surtout due au développement d'Aulacoseira subarctica (fig. 7) qui atteint son maximum en décembre alors que la concentration en $\mathrm{SiO}_{2}$ est maximale $\left(2 \mathrm{mg} . \mathrm{I}^{-1}\right)$. La croissance d'Asterionella formosa commence début février alors que Aulacoseira subarctica est en plein déclin; la concentration en $\mathrm{SiO}_{2}$ est alors de l'ordre de $1 \mathrm{mg} . \mathrm{l}^{-1}$. Dans d'autres lacs du Massif central, par exemple le lac Pavin, il faut remarquer que cette dernière espèce domine le phytoplancton au printemps (Amblard 1988, Amblard et Bourdier 1990).

II faut observer que certains groupes ou genres ou même espèces, même si cela ne se traduit pas au travers des valeurs de la biomasse, ont également leur maximum annuel lors du pic printanier. C'est le cas, par exemple, du genre Cryptomonas sensu Bourrelly 1985 (Cryptophycées Pyrrhophytes). Pendant un cours moment (mars 1987), ce genre dépasse Asterionella formosa en densité cellulaire et il représente alors $20 \%$ de la biomasse phytoplanctonique (fig. 6).

A la fin du printemps, le processus de sédimentation des diatomées précède de peu l'établissement de la 
stratification thermique. Approfondissement de la zone euphotique et stratification correspondent au minimum de biomasse mais s'accompagnent d'une diversification du peuplement algal. Pendant toute cette période, on a affaire à une biocénose instable dont les taxons dominants (en densité cellulaire ou en biomasse) peuvent changer rapidement. Ces fluctuations sont dues d'une part à la croissance momentanée de taxons type Dinobryon ou d'espèces de petite taille à rapport S/V élevé (type Dictyosphaerium pulchellum Wood) et d'autre part au développement tout aussi transitoire de Gonyostomum semen (Le Cohu et al. 1989). La présence de cette algue constitue une des originalités du réservoir de Pareloup, premier lac français où cette espèce a été observée en grande quantité (sa présence avait été remarquée en 1981, N. Lair, comm. pers.). En 1987, les deux pics estivaux de biomasse (fig. 2) sont dus à deux phases de croissance de cette espèce qui constitue alors $70 \%$ de la biomasse phytoplanctonique (fig. 6).

Dans les deux cas, le maximum de population se concentre au-dessous de $5 \mathrm{~m}$ de profondeur et cela se répercute de façon spectaculaire comme nous l'avons déjà signalé, sur les profils verticaux de la chlorophylle a (fig. 5). Gonyostomum semen est une espèce qui fuit les fortes intensités lumineuses, puisque, au milieu de la journée, les maximums de population se placent dans une zone où les intensités lumineuses sont inférieures à $90 \mu \mathrm{E} \cdot \mathrm{m}^{-2} \cdot \mathrm{s}^{-1}$ alors qu'en surface elles sont supérieures à $600 \mu \mathrm{E} \cdot \mathrm{m}^{-2} \cdot \mathrm{s}^{-1}$.
Le brassage automnal des eaux peut être marqué par le développement, relativement important en densité cellulaire, d'espèces de petite taille (Ochromonas sp., Chrysophycées ou Merismopedia tenuissima, Cyanophycées), le retour des diatomées et la part conséquente prise par Gonyostomum semen dans les valeurs de la biomasse. II faut remarquer que, pendant cette période d'isothermie automnale, la population de Gonyostomum semen représente entre 40 et $60 \%$ de la biomasse (fig. 6) avec une distribution quasi homogène sur toute la colonne d'eau. La stratégie adaptative de Gonyostomum semen, dans la mesure où l'intensité lumineuse est faible, couvre donc des conditions très différentes (stratification et isothermie). Pendant la période estivale les poussées de croissance de Gonyostomum semen aboutissent à un pic suivi d'une chute brutale, de l'ordre d'une semaine. Dans cet espace de temps, les conditions du milieu n'ont jamais évolué d'une manière drastique; on peut donc penser que la prédation intervient de manière prépondérante dans ce déclin; Gonyostomum semen est consommé par certains rotifères (Cronberg et al. 1988) et sans doute par les gardons. En octobre, novembre et décembre (1991), avec l'isothermie, les conditions hydrodynamiques changent considérablement, on peut penser que l'impact de la prédation est moins fort, la population de Gonyostomum semen étant beaucoup plus dispersée. Actuellement, les besoins nutritifs de cette espèce demandent encore à être précisés. 
Un caractère commun réunit tous les lacs où un développement important a été observé : ce sont tous des lacs aux eaux faiblement tamponnées, donc à faibles concentrations en calcium (Cronberg et al. 1988), ce qui est le cas du réservoir de Pareloup (\#10 mg..$^{-1}$ ). Grâce à sa mobilité en période de stratification et à ses faibles exigences vis à vis de la lumière, cette espèce semble pouvoir s'adapter à tous les milieux plus ou moins perturbés par l'homme à condition que les concentrations en calcium restent faibles. II ne faut pas oublier qu'à l'origine cette espèce a été décrite dans les tourbières à sphaignes (Bourrelly 1985). La question est également de savoir si cette algue, notamment pendant la stratification, n'est pas capable de tirer une substantielle fraction de son énergie et des nutriments qui lui sont nécessaires en ingérant des bactéries, comme cela a déjà été montré pour d'autres algues flagellées (Porter et al. 1987, Kimura et Ishida 1985, Bird et Kalff 1986 et 1987).

\section{DISCUSSION}

Le réservoir de Pareloup se caractérise par une biomasse phytoplanctonique minimale (poids frais en $\mu \mathrm{g}$ ) pendant la période de stratification et maximale fin de l'hiver-printemps. Fait important à remarquer, ce maximum est précédé d'une phase de croissance qui débute dès le mois de novembre. Dans un premier temps, cette phase de croissance de la biomasse est liée au développement d'Aulaco- seira subarctica (fig. 7) avec un maximum en décembre (mais pas de prélèvement en janvier); il faut d'ailleurs remarquer que, même à cette époque, Gonyostomum semen peut représenter jusqu'à $25 \%$ de la biomasse (1991). A partir de février, Asterionella formosa prend le relais et constitue la quasi totalité de la biomasse, son déclin étant provoqué par une carence en silice.

Un autre fait important dans le fonctionnement du réservoir de Pareloup doit être mis en exergue : la clarification de l'eau avec un doublement de la profondeur de la zone euphotique est due uniquement à la sédimentation des diatomées. La prédation n'y joue aucun rôle contrairement à ce qui est décrit dans le modèle proposé par Sommer et al. (1986). Dans ce modèle, l'évolution saisonnière du peuplement phytoplanctonique, du printemps à l'automne, est déterminée par des facteurs biologiques (compétition, broutage) et chimiques (déficience en éléments nutritifs); les facteurs physiques, lumière, température et brassage ne deviennent prépondérants qu'en automne et en hiver. Dans le réservoir de Pareloup, les quatre premières phases du modèle P.E.G. (Sommer et al. 1986) se réduisent à une seule, dominée par Asterionella formosa. La présence d'algues susceptibles d'être consommées par le zooplancton tel le genre Cryptomonas (phase 1 du modèle P.E.G.), atteignant son maximum au printemps, est en quelque sorte gommée. L'établissement de la stratification thermique correspondrait ici à la 
fin de la succession autogénique sensu Reynolds (1984).

Pendant toute la période de stratification, la croissance algale n'est limitée ni par l'azote ni par le phosphore (Thébault et Salençon 1993) : maximum de production primaire (Capblancq et al. 1988) et faible valeur globale de la biomasse (fig. 2 et 3) caractérisent toute cette période. Une autre caractéristique de cette biomasse algale est une grande hétérogénéité verticale de la densité cellulaire et des concentrations en chlorophylle a (Le Cohu et al. 1991).

Le modèle P.E.G. (Sommer et al. 1986) peut difficilement s'appliquer au réservoir de Pareloup qui s'en distingue par plusieurs particularités qui nous ont amenés à proposer une nouvelle approche de la succession phytoplanctonique :

1) La phase claire est induite par la sédimentation des diatomées et se prolonge tout l'été.

2) La mise en place de la stratification thermique est un facteur fondamental dans la mesure où se crée un milieu carencé en silice et un faible rapport Si/P.T.; le stade 11 du modèle P.E.G. avec la croissance de grandes diatomées ne peut se réaliser. Les espèces à rapport $S N$ élevé sont favorisées mais leur développement est contrôlé par la prédation (Salençon et Thébault 1994) et n'induit pas une forte biomasse.

3) La présence de Gonyostomum semen, se traduisant souvent de fa- çon importante dans la biomasse, donne un caractère original à la retenue de Pareloup (Le Cohu et al. 1989).

4) Dans le réservoir de Pareloup, si l'on souscrit au concept de succession phytoplanctonique proposé par Reynolds (1984) et repris par Amblard (1987), l'établissement de la stratification, comme nous l'avons déjà signalé, provoque la fin de la succession autogénique proprement dite avec un retour vers des stades pionniers au sein du biotope. Cette succession autogénique est donc interrompue par une perturbation allogénique (augmentation de la température de l'air) qui change la direction de la succession avec apparition d'un milieu (zone euphotique) seulement carencé en silice. Dans le réservoir de Pareloup l'établissement de la stratification thermique n'est pas un élément aléatoire, imprévisible, c'est au contraire un élément fondamental du fonctionnement du lac; la succession est alors de type cyclique, tel que le définit Ramade (1984) : démarrage de la succession au début de la stratification et stade ultime au printemps avec le maximum de biomasse. Dans la succession phytoplanctonique cyclique, la lumière est considérée comme une ressource au même titre que les autres facteurs nécessaires à la croissance des algues; la notion de saison n'entre pas en ligne de compte comme le montre l'augmentation progressive de la biomasse pratiquement à partir du brassage automnal. Dans cette succession cyclique quatre stades peuvent être distingués : 
1) période de stratification estivale avec une grande diversité du peuplement algal, chaque population ayant une croissance fugace (espèces en général à rapport $\mathrm{S} N$ élevé à l'exception de Gonyostomum semen);

2) période automnale de brassage avec présence de Gonyostomum semen et début de croissance de plusieurs populations de diatomées: Aulacoseira subarctica et ambigua, Cyclotella radiosa et Asterionella formosa;

3) période hivernale: Aulacoseira subarctica domine nettement, Gonyostomum semen présent;

4) fin de l'hiver et début du printemps : Asterionella formosa dominatrice et maximum de biomasse.

II faut remarquer que Gonyostomum semen intervient dans trois phases sur quatre: été, automne et même hiver.

\section{RÉFÉRENCES BIBLIOGRAPHIQUES}

Amblard C., 1987. Les successions phytoplanctoniques en milieu lacustre. Ann. Biol., XXI : 1-34.

Amblard C., 1988. Seasonal succession and strategies of phytoplankton development in two lakes of different trophic states. J. Plankton Res., 10 (6) : 1189-1208.

Amblard C. \& Bourdier G., 1990. The spring bloom of the diatom Melosira italica subsp. subarctica in lake Pavin : biochemical, energetic and metabolic aspects during sedimentation. $J$. Plankton Res., 12 (3) : 645-690.
Bird D.F. \& Kalff J., 1986. Bacterial grazing by planktonic lake algae. Sciences, 231: 493-495.

Bird D.F. \& Kalff J., 1987. Algal phagotrophy: Regulating factors and importance relative to photosynthesis in Dinobryon (Chrysophyceae). Limnol. Oceanogr., 32 : 277-284.

Bourrelly P., 1985. Les algues d'eau douce. III. Editions N. Boubée et Cie, Paris : $606 \mathrm{pp}$.

Capblancq J., Thébault J.-M. \& Jrad A., 1988. Relations entre la lumière et la photosynthèse dans un réservoir mésotrophe (Pareloup) : variations saisonnières des paramètres. Annls Limnol., 24 (1) : 39-48.

Cronberg G., Lindmark G. \& Björk S.,1988. Mass development of the flagellate Gonyostomum semen (Raphidophyta) in Swedish forest lake an effect of acidification?. Hydrobiologia, $161: 217-236$

Devaux J., 1973. Contribution à l'étude des populations phytoplanctoniques du Lac de Tazenat (Puy-de-Dóme). Ann. Stat. Biol. Besse-en-Chandesse, 7: 1-101.

Devaux J., 1977. Dynamique des populations phytoplanctoniques dans deux lacs du Massif central français. Ann. Stat. Biol. Besse-en-Chandesse, 10 : 1-184.

Falkowski P.G., 1984. Physiological responses of phytoplankton to natural light regimes. J. Plankton Res., 6 : 295-307.

Hoepffner N., 1984. Stratégies d'adaptation photosynthétique chez les diatomées de l'océan Antarctique: variations du nombre et de la taille des unités photosynthétiques. J. Plankton Res., 6 (5) : 881-895.

Jrad A., 1989. Phytoplancton et production primaire dans le lac de Pareloup (Aveyron). Thèse de doctorat $3^{\text {eme }} \mathrm{cy}$ cle. Toulouse : $166 \mathrm{pp}$.

Kimura B. \& Ishida Y., 1985. Photophagotrophy in Uroglena americana, Chryso- 
phyceae. Jap. J. Limnology, $46: 315-$ 318.

Le Cohu R., Guitard J., Comoy N. \& Brabet J., 1989. Gonyostomum semen (Raphidophycées), nuisance potentielle des grands réservoirs français? L'exemple du lac de Pareloup. Arch. Hydrobiol., 117 (2) : 225-236.

Le Cohu R., Comoy N., Guitard J. \& Brabet J., 1991. Périodicité du phytoplancton dans un réservoir de moyenne profondeur: le lac de Pareloup (Massif central, France), un exemple de succession cyclique. Annls. Limnol., 27 (3) : 197-214.

Lund J.W.G., Kipling C. \& Le Cren E.D., 1958. The inverted microscope method of estimating algal numbers and the statistical bases of estimations by counting. Hydrobiologia, 11 : 143-170.

Porter K.G., Sherr E.B., Sherr B.F., Pace M. \& Sanders W., 1985. Protozoa in planktonic food webbs. J. Protozool., 32 : 409-415.
Ramade F., 1984. Eléments d'écologie. Mc Graw Hill, Paris : 403 pp.

Reynolds C.S., 1984. The ecology of freshwater phytoplankton. Cambridge University Press : 384 pp.

Salençon M.J. et Thébault J.M., 1994. Modélisation de l'écosystème du Lac de Pareloup avec les modèles ASTER et MELODIA. Hydroécol. Appl. 6(1/2) : pp. 369-426.

Sommer U., Gliwicz Z.M., Lampert W. et Duncan A., 1986. The PEG-model of seasonal succession of planktonic events in fresh waters. Arch. Hydrobiol., 106 (4) : 433-471.

Sommer U., 1986. The periodicity of phytoplankton in Lake Constance (Bodensce) in comparison to other deep lakes of central Europe. Hydrobiologia, $138: 1-7$.

Thébault J.M. \& Salençon M.J., 1993. Simulation model of a mesotrophic reservoir (Lac de Pareloup, France): biological model. Ecol. Modelling, 65 : 1-30. 